\title{
Plan Agregado de Producción y la Productividad en una Planta de Producción de Conservas de Pescado
}

\author{
Aggregate Production Plan and Productivity in a Production Plant of Canned Fish \\ Giancarlos J. López Márquez¹, Joél A. Solis Sifuentes", Jaime E. Gutiérrez Ascón¹, Eulogio N. Morales Farías²
}

\section{RESUMEN}

Objetivo: Medir la relación que existe entre el plan agregado de producción, y la productividad del proceso de producción de conservas de pescado en la empresa Panafoods S.A.C. 2014. Métodos: La población fue de 311 trabajadores y la muestra ajustada de 115 al 95\% de nivel de confianza. Se hizo un análisis preliminar de Pareto, así como el método multiplicativo de Holt Winters, para el pronóstico del año 2014; índices de inventarios; índices de fuerza de trabajo; planeación agregada con el programa WinQSB 2.0, para seleccionar el plan óptimo y realizar el cálculo de la productividad. Resultados: el pronóstico de 616061 cajas, el costo de mantenimiento de inventario de 1,23 soles/caja, el requerimiento de 2,31 horas/caja, S/. 6,19 como costo hora normal, la hora extra a S/.7,74, costo de contratar a S/. 181,72 y de despedir a S/. 32,84; el plan agregado con la estrategia de tiempo constante de capacidad para empleados, en el que se cumple al $100 \%$ el pronóstico con un costo de S/. 9553 235.66; la productividad fue 0,065 cajas/soles; el modelo matemático que explica la investigación es: $Y=6,99 \times 10^{-2}-1,32 \times 10^{-9}\left(X_{1}\right)+8,53 \times 10^{-10}\left(X_{2}\right)+5,06 \times 10^{-7}\left(X_{3}\right)-8,18 \times 10^{-8}\left(X_{4}\right)$ con $r=99,9 \%$ (coeficiente de correlación). Conclusiones: El plan agregado de producción, se relaciona con la productividad del proceso de producción de conservas de pescado en la empresa Panafoods S.A.C. 2014. El presente estudio permitirá optimizar los recursos productivos, de manera que el incremento de productividad, con un ahorro en costos permita acciones de reinversión y mejora de procesos.

Palabras clave: Plan Agregado, productividad, pronóstico, inventario, estrategia.

\section{ABSTRACT}

Objective: Measure the relationship between aggregate production plan and productivity of the production process of canned fish in the Panafoods Company S.A.C. 2014. Methods: TThe population was 311 workers and the adjusted sample was 115 to $95 \%$ confidence level. A preliminary Pareto's analysis and Holt Winters multiplicative method for the 2014 prognosis was made; inventory rating; rates of work force; aggregate planning with Win QSB 2.0 program, to select the optimal plan and perform the calculation of productivity. Results: the forecast of 616.061 boxes, the cost of maintaining inventory of 1.23 soles / box, the requirement of 2.31 hours / box S/. 6.19 as timekeeping cost, the extra hour to S/. 7.74 , the cost of hiring to S/.181.72 and dismiss to S/. 32.84 ; the aggregate plan with the constant time strategy of capacity for employees, in which meets to $100 \%$ the forecast with a cost of S/. 9553235.66 ; the productivity was 0,065 boxes / soles; the mathematical model that explains the research is: $Y=6.99 \times 10-2-1.32 \times 10-9(X 1)+8.53 \times 10-10(X 2)+5.06 \times 10-7(X 3)-8.18 \times 10-8(X 4)$ with $r=99.9 \%$ (correlation coefficient). Conclusions: The aggregate production plan relates with the productivity of the production process of canned fish in Panafoods Company S.A.C. 2014. This study will allow to optimize the production resources, so that increased productivity, with a cost savings allow reinvestment actions and process improvement.

Keywords: Aggregate plan, productivity, forecast, inventory, strategy.

\footnotetext{
Facultad de Ingeniería Industrial, Sistemas e Informática. Universidad Nacional José Faustino Sánchez Carrión. Huacho, Perú.

${ }^{2}$ Facultad de Ciencias. Universidad Nacional José Faustino Sánchez Carrión. Huacho, Perú
} 


\section{INTRODUCCIÓN}

Los mercados internacionales han crecido tanto en oferta como en demanda, lo cual ha tenido un impacto en la adopción y ejecución de los procesos de planificación de la producción. Los mercados nacionales a partir de bloques comerciales como el Mercado Común Europeo, el Mercado Común del Sur (MERCOSUR), el Mercado Andino y otros continuarán expandiéndose. Es un imperativo competitivo adoptar y adaptar procesos de planificación de la producción enfocado a soportar este vasto ambiente. La empresa Pacific Natural Foods S.A.C. (Panafoods S.A.C.) no está exenta a estos cambios de mercado; de ahí que surge la discusión en torno a la productividad, siendo necesario encontrar las causas que la deterioran. En el caso de Panafoods S.A.C. el objetivo es la producción de conservas de pescado a un menor costo, a través del manejo adecuado de recursos productivos clave: como la fuerza de trabajo y los inventarios en función a la proyección de la demanda. Para esto deben enfocar esfuerzos para aumentar los índices de productividad actual y, en esa forma, reducir los costos de producción. Por ello, surge la necesidad de desarrollar planes que minimicen los costos relacionados con la variación de fuerza de trabajo y los niveles de inventarios, para mejorar la productividad. La falta de planeación agregada dentro de cualquier tipo de empresa genera una inadecuada organización de la elaboración del producto y/o servicio, así como la falta de control de los días a trabajar, ya que el personal tiene la necesidad de trabajar en ocasiones, fuera de las horas regulares (8 horas/día) de lunes a viernes, y termina haciéndolo muchas veces sábados y domingos, con el objetivo de entregar el producto a tiempo y sin contemplar los costos que lleva el realizar dicho producto lo que incide en la baja productividad de la empresa (Molina, 2013) (Herrera, 2010).

Se planteó la hipótesis que el plan agregado de producción, se relaciona con la productividad del proceso de producción de conservas de pescado en la empresa Panafoods S.A.C. 2014.

La presente investigación tuvo por objetivo medir la relación que existe entre el plan agregado de producción, y la productividad del proceso de producción de conservas de pescado en la empresa Panafoods S.A.C. 2014.

\section{MATERIAL Y MÉTODOS}

Para el plan agregado se consideró trabajar con una población de 311 operarios; se realizó un análisis preliminar a fin de evaluar las operaciones de la empresa usando el análisis de Pareto para determinar los productos vitales y luego clasificarlos por familias de productos. La proyección de la demanda para el año 2014 se calculó según Heizer y Render (2009) utilizando el método multiplicativo de Holt Winters con los datos de producción del año 2013.

Los índices de inventario se calcularon con la fórmula siguiente:

$$
C_{i}=\frac{C_{a}+C_{o}+C_{m e}}{\text { Ubicaciones }}
$$

Donde: el costo de mantener inventario $\left(C_{i}\right)$ es igual a la suma del costo del almacén $\left(C_{a}\right)$, costo operativo $\left(C_{\mathrm{o}}\right)$ y costo de máquinas y equipos $\left(C_{\mathrm{me}}\right)$ entre las ubicaciones totales del almacén. Según Cruelles 2013) se determinaron los índices de fuerza de trabajo como el costo de hora - hombre a tiempo normal y a tiempo extra, costo de contratar y despedir, y el tiempo requerido por unidad de producto a través de un estudio de tiempos y movimientos. Todos estos datos se emplearon para simular las estrategias de nivel en el programa WinQSB 2.0 con el sub programa Aggregate Planning (planeación agregada) donde analizamos 5 diferentes estrategias de nivel a fin de encontrar la más económica y que cumpla con la demanda, luego de esto se tabularon los datos del plan óptimo en hoja de cálculo para poder medir la 
productividad de cada mes del periodo de planeación (1 año) con la siguiente fórmula:

$$
\text { Productividad }=\frac{\text { Resultados Logrados (Cajas) }}{\text { Recursos Empleados (Soles) }}
$$

Luego se construyó un instrumento de 50 ítems aplicado a los dueños del problema (muestrade 115 trabajadores) elegidos aleatoriamente, instrumento que se validó por juicio de expertos (4 expertos) y se calculó la fiabilidad interna con alpha de Cronbach (Córdova 2012). Por último para medir la relación entre las variables plan agregado y productividad se realizó el modelo de regresión múltiple en hojas de cálculo con el programa XLStat - Pro v 7,52; la contrastación de hipótesis se realizó con la prueba de chi cuadrado.

\section{RESULTADOS}

El análisis preliminar, de los 30 productos elaborados en el año 2013, identificó 4 productos vitales mediante el análisis de Pareto, bajo el criterio 80/20; estos fueron: Filete de caballa en aceite vegetal $1 / 2 \mathrm{lb}$. (AFCO $1 / 2$ ), Grated de anchoveta en agua y sal $1 / 2 \mathrm{lb}$. (AGSA $1 / 2$ ), Grated de caballa en aceite vegetal $1 \mathrm{lb}$. Tall (AGCO Tall) y Grated de caballa en agua y sal $1 / 2 \mathrm{lb}$ (AGCA $1 / 2$ ), los que fueron clasificados en 3 familias de productos: Filete $1 / 2$, Grated $1 / 2$ y Grated Tall. (Figura 1 ).

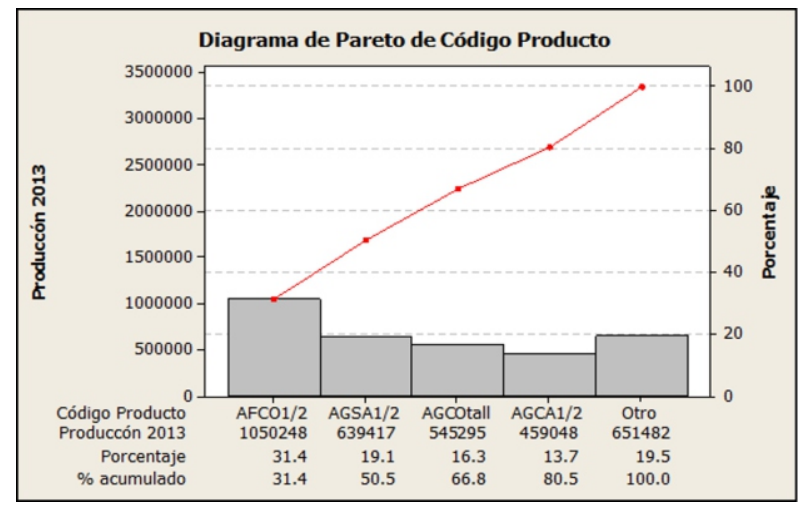

Figura 1. Análisis de Pareto de los 30 productos
Para la proyección de la demanda del año 2014, según Chase y Jacobs (2009) se utilizó el método multiplicativo de Holt Winters, desarrollándose ocho iteraciones; mediante las series de tiempo se analizó el año 2013, para determinar el modelo de pronóstico más exacto. Este pronóstico tiene un error porcentual absoluto (MAPE) de $6 \%$ y una desviación media absoluta (MAD) de 1839 cajas de conserva (Figura 2).

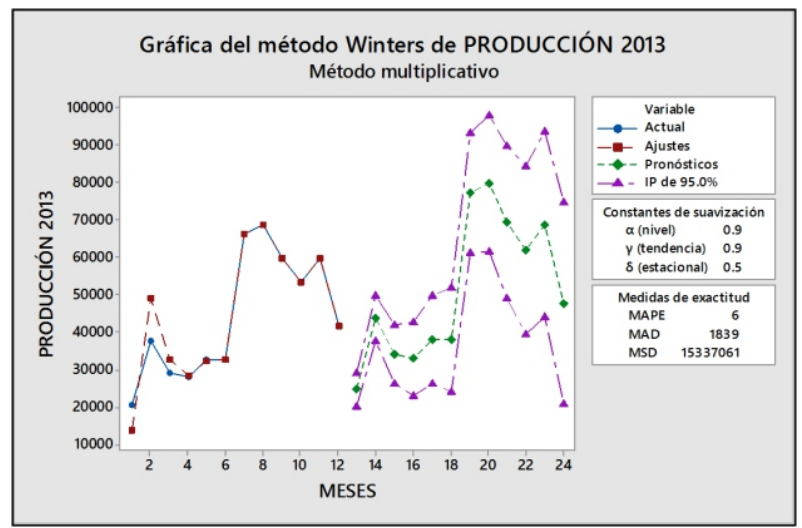

Figura 2. Pronóstico (método multiplicativo de Winters)

Con los índices de inventarios se determinó el costo mensual de mantener una unidad en inventario igual a 1,23 soles/caja; en base a una capacidad de almacenamiento de 152 ubicaciones (pallets standard), costo mensual de S/. 11867,67 por espacio de almacenamiento, S/. 12 150,00 por costo mensual de operación, costo mensual de S/.1 844,18 por concepto de máquinas y equipos, (formula 1).

Los índices de fuerza de trabajo determinaron la disponibilidad de 5584 horas laborables para el año 2014, el costo de 6,19 soles/H-h en tiempo normal y 7,74 soles/H-h en tiempo extra, S/. 181,72 el costo por contratar, S/. 32,84 por despedir, y el tiempo requerido por unidad de 2,31 horas/caja; estos datos de entrada que fueron ingresados al WinQSB, se obtuvieron con el programa Minitab 17, sirvieron como entrada para el plan agregado (Tabla 1 ). 
Tabla 1. Información de Plan Agregado (año 2014)

\begin{tabular}{lrrrrrrrrrrrr}
\hline \multicolumn{1}{c}{ Información (Inputs) } & ENE & FEB & MAR & ABR & MAY & JUN & JUL & AGO & SET & OCT & NOV & DIC \\
\hline Pronostico de la demanda (cajas/mes) & 24628 & 43736 & 34066 & 32879 & 37935 & 37961 & 77193 & 79702 & 69404 & 61905 & 68863 & 47791 \\
Numero inicial de empleados & 311 & & & & & & & & & & & \\
Tiempo regular disponible & 496 & 448 & 496 & 480 & 464 & 464 & 464 & 496 & 480 & 496 & 480 & 320 \\
Costo regular de la hora (SI.) & 6,19 & 6,19 & 6,19 & 6,19 & 6,19 & 6,19 & 6,19 & 6,19 & 6,19 & 6,19 & 6,19 & 6,19 \\
Costo por debajo de la hora (S/.) & 6,19 & 6,19 & 6,19 & 6,19 & 6,19 & 6,19 & 6,19 & 6,19 & 6,19 & 6,19 & 6,19 & 6,19 \\
Tiempo extra disponible & 4 & 4 & 4 & 4 & 4 & 4 & 4 & 4 & 4 & 4 & 4 & 4 \\
Costo de tiempo extra & 7,74 & 7,74 & 7,74 & 7,74 & 7,74 & 7,74 & 7,74 & 7,74 & 7,74 & 7,74 & 7,74 & 7,74 \\
Costo de contratación & 181,72 & 181,72 & 181,72 & 181,72 & 181,72 & 181,72 & 181,72 & 181,72 & 181,72 & 181,72 & 181,72 & 181,72 \\
Costo de despido & 32,84 & 32,84 & 32,84 & 32,84 & 32,84 & 32,84 & 32,84 & 32,84 & 32,84 & 32,84 & 32,84 & 32,84 \\
Inventario Inicial & 0 & 0 & 0 & 0 & 0 & 0 & 0 & 0 & 0 & 0 & 0 & 0 \\
Nivel máximo de inventario & $\mathrm{M}$ & $\mathrm{M}$ & $\mathrm{M}$ & $\mathrm{M}$ & $\mathrm{M}$ & $\mathrm{M}$ & $\mathrm{M}$ & $\mathrm{M}$ & $\mathrm{M}$ & $\mathrm{M}$ & $\mathrm{M}$ & $\mathrm{M}$ \\
Nivel mínimo de inventario aceptado & 0 & 0 & 0 & 0 & 0 & 0 & 0 & 0 & 0 & 0 & 0 & 0 \\
Costo de mantener una unidad en inventario & 1,23 & 1,23 & 1,23 & 1,23 & 1,23 & 1,23 & 1,23 & 1,23 & 1,23 & 1,23 & 1,23 & 1,23 \\
Otros costos unitarios de producción & 0 & 0 & 0 & 0 & 0 & 0 & 0 & 0 & 0 & 0 & 0 & 0 \\
Requerimiento de capacidad (hora/caja) & 2,31 & 2,31 & 2,31 & 2,31 & 2,31 & 2,31 & 2,31 & 2,31 & 2,31 & 2,31 & 2,31 & 2,31 \\
\hline
\end{tabular}

El valor de inventario inicial fue cero, debido a que la empresa en estudio es de tercerización (maquiladora), por lo tanto es el cliente quien envía la materia prima y demás artículos de producción para un determinado lote de pedido; el nivel máximo de inventario es considerado infinito (M) por defecto del programa, también permite un valor numérico, debiendo calcularse de acuerdo a las condiciones del almacén. Al procesar estos datos, el software permite evaluar 10 diferentes estrategias, debiendo simular las que se adecuen a las características de la producción; para el presente estudio se simuló con 5 diferentes estrategias de nivel (Tabla 2).

Al evaluar estas estrategias se analizan los factores de cumplimiento de demanda y costos incurridos, de tal manera que la decisión óptima sea la estrategia más económica y que más cerca este a cumplir la demanda (Gido 2001). En ese sentido, la mejor estrategia es Tiempo constante de capacidad para empleados, debido a que cumple con la demanda pronosticada en $100,06 \%$, a un costo de S/. 9 553 235,66 nuevos soles; no obstante que la estrategia cantidad mínima de empleados constantes tiene un costo mucho menor que asciende a S/. 4259 862,94, con un déficit de cumplimiento de demanda de $48,26 \%$, influiría significativamente en la productividad de la empresa Según la estrategia seleccionada se debe despedir 56 operarios, en la práctica esto no aplica porque solo se ha evaluado 4 de 30 productos que más contribuyen en las utilidades de la empresa, debiendo disponer de estos operarios para sumarse al trabajo de los demás productos (Tabla 3 ).

Tabla 2. Estrategias de Nivel del Plan Agregado

\begin{tabular}{lrrrrrrr}
\hline \multicolumn{1}{c}{ Estrategias de nivel } & \multicolumn{5}{c}{ Factores de análisis } \\
\cline { 2 - 8 } & $\begin{array}{l}\text { Cumplimiento } \\
\text { de demanda }\end{array}$ & $\begin{array}{c}\text { Costo Tiempo } \\
\text { Normal }\end{array}$ & $\begin{array}{c}\text { Costo Antes } \\
\text { de Tiempo }\end{array}$ & $\begin{array}{c}\text { Costo } \\
\text { Mantener } \\
\text { Inventario }\end{array}$ & $\begin{array}{c}\text { Costo de Costo Total del } \\
\text { Despedir }\end{array}$ \\
Plan
\end{tabular}


Tabla 3. Resultado del plan agregado de producción, Panafoods S.A.C. - año 2014

\begin{tabular}{|c|c|c|c|c|c|c|c|c|}
\hline Periodos & $\begin{array}{c}\text { Demanda } \\
\text { (cajas) }\end{array}$ & $\begin{array}{l}\text { Producción } \\
\text { Regular } \\
\text { (cajas) }\end{array}$ & $\begin{array}{l}\text { Producción } \\
\text { en sobre } \\
\text { tiempo } \\
\text { (cajas) }\end{array}$ & $\begin{array}{c}\text { Producción } \\
\text { total } \\
\text { (cajas) }\end{array}$ & $\begin{array}{l}\text { Inventario } \\
\text { Final } \\
\text { (cajas) }\end{array}$ & $\begin{array}{c}\text { Contratar } \\
\text { (empleados) }\end{array}$ & $\begin{array}{c}\text { Despedir } \\
\text { (empleados) }\end{array}$ & $\begin{array}{l}\text { Numero de } \\
\text { Empleados }\end{array}$ \\
\hline Inicial & & & & & 0 & & & 311 \\
\hline Enero & 24628 & 54753 & 0 & 54753 & 30125 & 0 & 56 & 255 \\
\hline Febrero & 43736 & 49455 & 0 & 49455 & 35844 & 0 & 0 & 255 \\
\hline Marzo & 34066 & 54753 & 0 & 54753 & 56531 & 0 & 0 & 255 \\
\hline Abril & 32879 & 52987 & 0 & 52987 & 76639 & 0 & 0 & 255 \\
\hline Mayo & 37935 & 51221 & 0 & 51221 & 89925 & 0 & 0 & 255 \\
\hline Junio & 37961 & 51221 & 0 & 51221 & 103185 & 0 & 0 & 255 \\
\hline Julio & 77193 & 51221 & 0 & 51221 & 77213 & 0 & 0 & 255 \\
\hline Agosto & 79702 & 54753 & 0 & 54753 & 52264 & 0 & 0 & 255 \\
\hline Setiembre & 69404 & 52987 & 0 & 52987 & 35847 & 0 & 0 & 255 \\
\hline Octubre & 61905 & 54753 & 0 & 54753 & 28695 & 0 & 0 & 255 \\
\hline Noviembre & 68863 & 52987 & 0 & 52987 & 12819 & 0 & 0 & 255 \\
\hline Diciembre & 47791 & 35325 & 0 & 35325 & 353 & 0 & 0 & 255 \\
\hline Total & 616063 & 616416 & 0 & 616416 & 599440 & 0 & 56 & \\
\hline
\end{tabular}

Tabla 4. Análisis de costos para el plan agregado de producción - Panafoods S.A.C. - año 2014

\begin{tabular}{lccccccc}
\hline Periodos & $\begin{array}{c}\text { Tiempo } \\
\text { normal (S/.) }\end{array}$ & $\begin{array}{c}\text { Antes de } \\
\text { tiempo(S/.) }\end{array}$ & $\begin{array}{c}\text { Sobre tiempo } \\
(\mathrm{S} / .)\end{array}$ & $\begin{array}{c}\text { Costo de } \\
\text { mantener } \\
\text { Inventario }\end{array}$ & $\begin{array}{c}\text { Contratar } \\
(\mathrm{S} / .)\end{array}$ & $\begin{array}{c}\text { Despedir } \\
(\mathrm{S} / .)\end{array}$ & $\begin{array}{c}\text { Costo Total } \\
(\mathrm{S} / .)\end{array}$ \\
\hline Enero & 782907,69 & 3,53 & 0 & 37053,75 & 0 & 1839,04 & 821804 \\
Febrero & 707152,06 & 0 & 0 & 44088,12 & 0 & 0 & 751240,19 \\
Marzo & 782907,69 & 3,53 & 0 & 69533,13 & 0 & 0 & 852444,91 \\
Abril & 757655,81 & 0,19 & 0 & 94265,97 & 0 & 0 & 851921,94 \\
Mayo & 732403,94 & 0 & 0 & 110607,75 & 0 & 0 & 843011,69 \\
Junio & 732403,94 & 0 & 0 & 126917,55 & 0 & 0 & 859321,50 \\
Julio & 732403,94 & 0 & 0 & 94971,99 & 0 & 0 & 827375,94 \\
Agosto & 782907,69 & 3,53 & 0 & 64284,72 & 0 & 0 & 847195,94 \\
Setiembre & 757655,81 & 0,19 & 0 & 44091,81 & 0 & 0 & 801747,81 \\
Octubre & 782907,69 & 3,53 & 0 & 35294,85 & 0 & 0 & 818206,06 \\
Noviembre & 757655,81 & 0,19 & 0 & 15767,37 & 0 & 0 & 773423,38 \\
Diciembre & 505108,66 & 0 & 0 & 434,19 & 0 & 0 & 505542,84 \\
Total & 8814071 & 14,7 & 0 & 737311,25 & 0 & 1839,04 & 9553236 \\
\hline
\end{tabular}

En la Tabla 4, el costo de tiempo normal se determinó del producto de cajas producidas, tiempo requerido por cajas y costo de hora normal (costo regular de la hora); el costo antes de tiempo, es el costo de las horas no utilizadas en la producción, es decir si se termina la producción antes de las horas disponibles se costea la diferencia en base al costo por debajo de la hora.
La productividad total para el período de planeación y la productividad mensual (enero a diciembre, 2014) en cajas/nuevos soles y con el fin de medir objetivamente la productividad del plan agregado de producción para la organización, se calculó utilizando la fórmula 2 , encontrándose una productividad total de 0,065 cajas/nuevo sol, de la misma forma se obtuvieron las productividades mensuales (Tabla 5).

Tabla 5. Productividades mensuales

\begin{tabular}{lllllllllllll}
\hline Periodos & Enero & Febrero & Marzo & Abril & Mayo & Junio & Julio & Agosto & Setiembre & Octubre & Noviembre & Diciembre \\
\hline $\begin{array}{l}\text { Productividad } \\
\text { (Cajas/soles) }\end{array}$ & 0,067 & 0,066 & 0,064 & 0,062 & 0,061 & 0,06 & 0,062 & 0,065 & 0,066 & 0,067 & 0,069 & 0,07 \\
\hline
\end{tabular}


Para determinar el modelo matemático de la investigación utilizamos el programa XLStatPro Versión 7.5.2., donde la función matemática que explica el comportamiento entre las variables plan agregado y productividad es la siguiente: $Y=6,99 \times 10^{-2}$ $1,32 \times 10^{-9}\left(X_{1}\right)+8,53 \times 10^{-10}\left(X_{2}\right)+5,06 \times 10^{-7}\left(X_{3}\right)-$ $8,18 \times 10^{-8}\left(X_{4}\right)$ obteniendo un coeficiente de correlación $r=99,9 \%$, lo cual indica que la relación entre las variables de esta investigación, es muy alta; por otro lado se obtuvo un coeficiente de determinación $\mathrm{R}^{2}=$ $99,8 \%$ lo cual explica el grado de variabilidad entre las variables en estudio. Donde:

$X^{1}=$ Proyección de la demanda, $X^{2}=$ Inventario, $X^{3}=$ Fuerza de Trabajo, $X^{4}=$ Estrategia de nivel.

En la contrastación de hipótesis se utilizaron los valores cualitativos del instrumento documental (Plan Agregado y Productividad) en las categorías comprendidas dentro de la escala valorativa de Likert, a fin de responder las hipótesis planteadas en esta investigación (Córdova 2012). Mediante la prueba de hipótesis chi cuadrado, con valores para $X^{2}$ Calculado $=22,665$ y X $X^{2}$ Crítico $=21,030$, se rechazó la hipótesis nula y se aceptó la hipótesis planteada, a un nivel de confianza del 95\%; es decir, que el plan agregado de producción, se relaciona con la productividad del proceso de producción de conservas de pescado en la empresa Panafoods S.A.C. 2014. El test chi cuadrado se eligió por las siguientes condiciones: la hipótesis debe tratar sobre relación o dependencia de variables, ambas deben ser cualitativas y la muestra puede ser de cualquier tamaño.

\section{DISCUSIÓN}

Dado que la productividad se relaciona directamente con los recursos empleados para alcanzar los resultados, de acuerdo a nuestro modelo general de la investigación: $\mathrm{Y}=$ $6,99 \times 10^{-2}-1,32 \times 10^{-9}$ (Proyección de la demanda $)+8,53 \times 10^{-10}($ Inventario $)+5,06 \times 10^{-7}$ (Fuerza de trabajo) - 8,18x10-8(Estrategias de nivel), encontramos que el inventario es el que influye en gran medida en la productividad con un coeficiente de correlación $r=99,9 \%$ lo que indica que la correlación entre el inventario y la productividad es muy alta, es decir el incremento o disminución de los costos de almacenamiento, el incremento o disminución del volumen de inventario en los almacenes influirá en la variación de la productividad de la empresa, lo cual está de acuerdo con Bonilla (2014), quien establece una clara relación entre la planeación de la producción y la productividad para evitar en el gasto excesivo de los recursos económicos. En la contrastación de hipótesis utilizando los valores cualitativos del instrumento documental (Plan Agregado y Productividad) en las categorías comprendidas dentro de la escala valorativa de Likert; mediante la prueba de hipótesis chi cuadrado, con valores para y, se rechazó la hipótesis nula y se aceptó la hipótesis planteada, a un nivel de confianza del 95\%;es decir, que el plan agregado de producción, se relaciona con la productividad del proceso de producción de conservas de pescado en la empresa Panafoods S.A.C. 2014, lo que concuerda con Herrera (2010), el cual sostiene que el diseño de una planeación agregada mejora las operaciones de la división de planeamiento y control de una empresa como la metalmecánica SIMA-Perú.

\section{REFERENCIAS BIBLIOGRÁFICAS}

Bonilla, E. (Julio de 2014). Planeación de la producción para el mejoramiento de la productividad de acoples y cápsulas en la Compañía Impofreico S. A. Recuperado el 25 de Agosto de 2014, de h t t p : / / re po.ut a.ed u. e c : http://repo.uta.edu.ec/handle/12345678 9/7773

Chase, R., Jacobs, R., \& Aquilano, N. (2009). Administración de Operaciones Producción y Cadena de Suministros (Duodécima ed.). México D.F.: MC Graw Hill.

Córdova, M. (2012). Estadística Aplicada a la Investigación Volumen I. Lima: Editorial San Marcos.

Cruelles, J. A. (2013). Ingeniería industrial Métodos de trabajo, tiempos y su aplicación a la planificación ya la mejora continua. México D.F.: Alfaomega.

Gido, J., \& Clements, J. (2001). Administración exitosa de proyectos. México, D.F.: THOMSOM Editores. 
Herrera, B. (2010). Diseño de una Planeación Agregada para la mejora de las operaciones de la División de Planeamiento y Control de la Producción de la Empresa Metalmecánica de Servicios Industriales de la Marina SIMA - Chimbote. Tésis para optar el Título de Ingeniero Industrial, Pimentel: Universidad César Vallejo.

Molina, C. (2013). Plan agregado de producción para el mejoramiento de la productividad de la Empresa Ecuatoriana de Curtidos S. A. Tesis para optar el Título de Ingeniería Industrial en Procesos de automatización, Ambato: Universidad Técnica de Ambato.

Correo electrónico: carlos20.lopez@gmail.com

Revisado por pares:

Recibido: 20-10-2015

Aceptado: 20-06-2016 